\title{
Investindo no Big Brother Brasil: uma análise da economia política de um marco da indústria midiática brasileira
}

\author{
Bruno Campanella 1 \\ UFRJ
}

\begin{abstract}
Resumo: Este trabalho analisa o reality show Big Brother Brasil sob a perspectiva da economia política. A sua exibição em diversas plataformas tais como TV aberta, TV paga, pay-per-view, internet e telefonia celular abre não somente novas possibilidades para sua exploração comercial, mas, principalmente, proporciona um importante posicionamento estratégico para a Rede Globo dentro do contexto da convergência midiática brasileira.

Palavras-chave: Big Brother Brasil, economia política, multi-plataforma, convergência.

Abstract: This work analyses the reality show Big Brother Brasil under a political economic perspective. Its multi-platform broadcasting via open $T V$, pay TV, pay-per-view, Internet and mobile phone not only opens new possibilities for its commercial exploration, but, crucially, presents Rede Globo an important strategic positioning within the Brazilian media convergence context.
\end{abstract}

Key words: Big Brother Brasil, political economy, multiplatform, convergence.

1 Bruno Campanella é doutorando em comunicação e cultura pela ECO-UFRJ. 


\section{Introdução}

Devido ao seu formato inovador, o programa de reality show Big Brother tem gerado inúmeras discussões dentro da academia acerca dos seus significados e impactos na sociedade. Enquanto alguns o consideram como um marco na televisão mundial pela sua capacidade de hibridizar diversos gêneros televisivos (CASTRO 2006), outros afirmam que o Big Brother mudou a própria cultura, ao tornar a performance da autenticidade uma característica chave da interação humana (ANDREJEVIC 2005). O interesse despertado é tal, que podemos achar trabalhos abordando o programa a partir de disciplinas tão diversas quanto a psicologia (BEATTIE 2003), os estudos de gênero (STARRS 2004), a lingüística (THORNBORROW \& MORRIS 2004), o teatro (LAVENDER 2003), e até mesmo a antropologia da religião (DUTTON 2006). Na área da comunicação, podemos encontrar inúmeros artigos e capítulos publicados sobre o Big Brother - incluindo uma edição inteiramente dedicada ao programa na revista acadêmica Television and New Media no ano de 2002 -, além da coletânea de ensaios editada por Ernest Mathijs e Janet Jones no livro Big Brother International, onde são investigadas versões do programa exibidas em diferentes países, e sob várias perspectivas. Isto sem mencionar os inúmeros livros e readers analisando o gênero dos reality shows, onde o Big Brother é freqüentemente citado com destaque.

De um modo geral, os trabalhos acadêmicos sobre o novo formato tentam entender este reality show sob a ótica dos estudos culturais e midiáticos. Algumas destas análises investigam as implicações da transmissão multi-plataforma ${ }^{2}$ no processo de recepção do programa (JONES 2004; ROSCOE 2004a; TINCKNELL \& RAGHURAM 2002), outras se preocupam em estudar as possibilidades abertas pela interatividade oferecida pelo Big Brother (CORNER 2002; HOLMES 2004). Também podemos encontrar trabalhos que problematizam o conceito de autenticidade junto aos fãs do reality show (ANDACHT 2004; HILL 2005; JONES 2003; VAN ZOONEN 2001), os que enxergam o formato como campo fértil de estudo da formação e legitimação das identidades contemporâneas (CHANDLER \& GRIFFITHS 2004;

\footnotetext{
${ }^{2} \mathrm{O}$ Big Brother é um produto multi-plataforma na medida em que ele existe em diferentes mídias, tais como televisão aberta, televisão paga, pay-per-view, internet e telefonia celular. O programa só consegue ser entendido em sua complexidade se analisado levando-se em consideração todas estas mídias.
} 
COULDRY 2002; PALMER 2002), além das análises que buscam no conceito de gênero, a chave para entender o sucesso deste 'evento midiático' (CASTRO 2006; JOST 2004).

Embora muitos autores reconheçam a importância mercadológica deste novo formato para a criação de fontes alternativas de receita (ANDREJEVIC 2005; BAZALGUETTE 2005; CARTER 2004), num mundo que caminha para a convergência das mídias (CASTRO 2006; MURDOCK 2006), nenhum deles tentou se aprofundar em uma discussão de cunho estritamente econômico político. Tanto Andrejevic quanto Carter percebem o Big Brother como um programa que ajuda na constituição de subjetividades consoantes com a nova economia virtual (ANDREJEVIC 2005; CARTER 2004). Também explorando a criação de novas subjetividades, Bratich afirma que as características do reality show em questão auxiliam na formação de sujeitos maleáveis e colaborativos, que se enquadram perfeitamente na sociedade contemporânea voltada ao mercado (BRATICH 2006).

Apesar de estes trabalhos se preocuparem com a relação de programas como o Big Brother com o sistema econômico atual, eles evitam fazer análises mais concretas sobre os processos produtivos dentro do contexto midiático de tais produtos. Talvez, o trabalho que mais se aproxime de tal objetivo seja a investigação dos processos de produção da edição sueca do Big Brother do ano de 2004, realizado por Sanne Ollila e Tobias Fredberg. Em seu estudo, Ollila e Fredberg optaram por focar em aspectos administrativos e micro-econômicos daquela edição do reality show. No entanto, eles não tentam dar uma visão macro do posicionamento estratégico do Big Brother para o Kanal 5 (responsável pela produção do programa na Suécia) ou mesmo do seu significado para o mercado televisivo de um modo geral (OLLILA \& FREDBERG 2004).

Por entender que este tipo de análise é fundamental se quisermos compreender de maneira mais ampla o sucesso da exportação deste formato para mais de 50 países ${ }^{3}$ pela Endemol, o presente texto se propõe a explorar o caso do Big Brother Brasil, analisando de que forma este programa ocupa uma posição estratégica não somente dentro da Rede Globo, empresa detentora dos seus direitos

${ }^{3}$ www.endemol.com 
no país, mas em todo o mercado nacional, principalmente se levarmos em consideração o breve início das operações da televisão digital no Brasil. Apesar de o presente foco ser na versão brasileira do programa, muitas observações podem servir de inspiração para se pensar o papel deste reality show em outros contextos nacionais.

A grande contribuição dada pelos diversos estudos que tentam compreender o fenômeno do Big Brother sob uma perspectiva culturalista, muitas vezes enfatizando sua capacidade de se adequar à cultura local onde é produzido (FRAUMEIGS 2006; MADGER 2004; MATHIJS \& JONES 2004; ROSCOE 2004b), não diminui a nossa crença na relevância de uma análise econômico/ política deste objeto. Como bem argumenta Chad Raphael em seu estudo da economia política do gênero de reality television nos Estados Unidos, tal perspectiva só pode complementar o entendimento das implicações deste programa dentro do contexto do atual sistema econômico capitalista no qual vivemos.

"Although my focus here is on political economy rather than textual or audience issues, I do not want to imply that these programs' cultural significance can be reduced to their relations of production and distribution. Yet without understanding the political-economic forces that drove the spread of these genre, textual and audience studies may risk reifying it as an expression of audience demand, or of their creators, or of a cultural, discursive, or ontological shift unrelated to the needs of those who run the television industry." (RAPHAEL 2004, p.119)

Assim sendo, como ponto de partida desta análise, nós vamos sugerir que o Big Brother Brasil têm a sua grande importância mercadológica devido a dois fatoreschave: a sua capacidade de explorar de maneira inovadora a convergência das suas diferentes mídias de transmissão (televisão aberta, televisão paga, pay-per-view, internet e telefonia celular), e o seu grande potencial de criação de novas fontes de receita para além da tradicional venda de espaço publicitário para televisão.

\section{Um programa Multi-Plataforma}

Em sua descrição das mudanças no consumo ao longo do século $\mathrm{XX}$, Graham Murdock associa os principais ambientes de comercialização de mercadoria de cada época à emergência de uma mídia predominante, assim como a um novo princípio. De acordo com essa categorização, as lojas de departamento estão 
associadas ao cinema, e ambos compartilham o princípio da exibição. Num momento seguinte, a televisão comercial passa a se destacar como mídia de maior importância, estando ela associada à popularização dos supermercados e à idéia de fluxo. Já na contemporaneidade, de acordo com Murdock, o consumo em massa está ligado principalmente aos mega malls e aos parques temáticos, que, por sua vez, acompanham o crescimento das mídias interativas. O princípio fundamental de nossa época é o da convergência (MURDOCK 2006).

Dentro desta tendência contemporânea, a convergência das mídias começou a ser adotada pelo grupo Globo como estratégia de mercado a partir dos anos 90. Naquela época, o grupo iniciou a diversificação de seus investimentos na área de comunicação, buscando se afirmar em toda a cadeia produtiva do setor de mídia, da produção à distribuição.

Junto com a Italia Telecom e o Banco Bradesco, a Globo montou a Maxtel, uma operação de telefonia celular nos estados da Bahia, Sergipe e Minas Gerais. Ela também se associou à Victori International para criar a Vicom, uma empresa de distribuição de dados. Dentro da área de televisão, a Globo se associou à SKY de Rupert Murdoch para lançar um sistema de transmissão por satélite em DTH. Porém, o maior investimento de todos, foi na operação de distribuição de televisão e internet à cabo, chamada de Globocabo (posteriormente rebatizada de NET). Este negócio, entretanto, dependia de dois fatores fundamentais para contrabalançar os pesados investimentos necessários para sua implantação: o rápido crescimento da base de assinantes, e a estabilidade do Real, uma vez que os custos, tanto financeiros (decorrentes de empréstimos no exterior) quanto de conteúdo eram em dólar. Todavia, os prognósticos dos executivos do grupo se mostraram demasiadamente otimistas. A base de assinantes não decolou, e o Real começou a se desvalorizar rapidamente em relação ao dólar. A situação chegou a uma situação crítica no final de 2002, obrigando tanto a Globopar, holding do grupo (excluindo a Rede Globo, o Infoglobo e o Sistema Globo de Rádio) quanto a NET a anunciar default ${ }^{4}$. A estratégia do grupo teve que ser redirecionada. Ele iria passar a se concentrar na produção de conteúdo, ao mesmo tempo em que começaria a se desfazer, ou pelo menos diminuir

\footnotetext{
4 Default é a situação que uma empresa chega quando não tem mais capacidade de pagar os seus compromissos financeiros. Ela se torna inadimplente.
} 
drasticamente, a participação em seus negócios de distribuição. Esta mudança também era fundamental para que o grupo Globo pudesse readquirir a confiança dos investidores (GINDRE 2005).

Independente dos percalços sofridos pela Globo nos últimos anos, o processo de convergência digital das mídias continua sendo uma prioridade. Entretanto, como o foco agora está na produção de conteúdo, a busca por canais alternativos para distribuí-lo passa a ser crucial para o grupo. Dentro deste contexto, o seu portal na internet Globo.com adquire uma importância fundamental, uma vez que, de acordo com Gindre, as organizações Globo apostam que

"a Internet estará, cada vez mais, disponível em vários equipamentos, além dos já tradicionais computadores. O portal "empacota" o conteúdo produzido pelas diversas unidades do grupo, prepara-os para serem disponibilizados na Internet e acessados por distintos terminais (PCs, notebooks, telefones celulares e, quem sabe, terminais de TV digital). Pela própria lógica da Internet, este material deve estar preparado para ser consumido por demanda, de acordo com necessidades e gostos do usuário." (GINDRE 2005, p.51)

O Big Brother, como nenhum outro programa de televisão, se encaixa perfeitamente neste novo ambiente multi-midiático. Ele é um produto feito para ser consumido tanto em TV aberta, TV paga, pay-per-view, internet, quanto por telefonia celular.

No Brasil, a Globo produz o programa junto à Endemol, mantendo uma parceria com a NET para a distribuição da versão ao vivo do reality show em payper-view, além de um acordo com a Vivo para o 'empacotamento' da versão em celular do programa (GINDRE 2005). A versão em TV paga é produzida pelo canal Multishow que é parte da programadora de canais Globosat, de propriedade do grupo 5 .

Entretanto, é justamente a sua versão na internet, com a sua capacidade de agregar um considerável volume de usuários para o seu site oficial, que talvez tenha mais a agregar ao grupo Globo.

\footnotetext{
5 É importante notar que o Big Brother, durante o seu período de produção, gera diversos programas agregados tanto na TV Globo, quanto no canal Multishow. Entre eles podemos destacar Só Para Maiores na Globo, e Eliminação BBB do Multishow.
} 
Desde a sua primeira exibição na Holanda em 1999, os produtores entenderam que esta nova mídia seria fundamental para prover uma experiência mais completa do reality show para os seus fãs. Apesar de sofrer variações dependendo do país em que o Big Brother é produzido, o site oficial costuma trazer informações, tais como, as regras do programa, o perfil dos participantes, atualizações sobre acontecimentos dentro da casa na forma de diários, fotografias e vídeos, salas e bate-papo para os fãs, blogs, votações para eliminação dos participantes e pesquisas com os fãs. Sem mencionar a possibilidade de acesso à versão ao vivo do programa ${ }^{6}$.

Peter Bazalgette, no livro onde narra a criação da Endemol, afirma que, mesmo a empresa tendo desenvolvido diversos formatos televisivos, foi o Big Brother quem chamou a atenção dos investidores do mercado financeiro e de empresas interessadas em investir em conteúdos capazes de convergir várias mídias, e acima de tudo, gerar acesso em seu site na internet. No início de 2000, o sucesso conquistado pela primeira edição do programa a ir ao ar na Holanda, tanto em termos de índices de audiência, acessos ao site oficial, e volume de ligações telefônicas, fez com que a Endemol, depois de uma disputa entre vários pretendentes, fosse vendida para o grupo Telefonica da Espanha por impressionantes $€_{5.5}$ bilhões de euros. Em um período de fartura nos mercados financeiros mundiais, a possibilidade de investir numa empresa com capacidade de produzir conteúdo televisivo que também pudesse ser consumido de maneira tão bem sucedida em outras mídias, especialmente a internet, parecia uma tentação grande demais para os investidores daquela época. John de Mol, o fundador principal da Endemol, conseguiu aproveitar o final do período da famosa 'bolha da internet' nos mercados financeiros mundiais como poucos (BAZALGUETTE 2005).

Tamanho entusiasmo não era sem motivos. No Reino Unido, por exemplo, a primeira versão do programa trouxe números surpreendentes. O canal Channel 4, responsável pela sua exibição naquele país, conseguiu quebrar o seu recorde histórico de share de audiência em uma sexta-feira, 46 por cento ( 9 milhões de pessoas). Quase 70 por cento da população britânica assistiu a pelo menos um episódio

\footnotetext{
${ }^{6}$ No Brasil, o site oficial do Big Brother, hospedado no portal Globo.com, oferece 2 câmeras exclusivas extras que não são disponibilisadas em pay-per-view.
} 
daquela edição do Big Brother. Mais de 7 milhões de pessoas votaram por telefone na final do programa, um recorde na história da televisão inglesa. Já o site oficial do Big Brother recebeu uma média de 3 milhões de acessos por dia durante a exibição do reality show, fazendo deste, o site com o maior número de acessos em toda Europa durante aquele período, independente do gênero (HILL 2005, p.32).

No Brasil não foi diferente. Certamente, o volume de acessos que o Big Brother traz para o portal Globo.com, onde ele fica hospedado, é uma das grandes apostas do grupo Globo. Estratégias bem sucedidas, como a vinculação da criação de uma conta de email no portal Globo.com para que se possa enviar uma fita de testes para participar do Big Brother, ou a possibilidade do fã do programa ter um link de seu blog selecionado para fazer parte do site oficial do reality show, desde que este blog esteja hospedado na ferramenta de blogs 8P - que faz parte da Globo.com -, demonstram uma preocupação do grupo em aproveitar a popularidade do programa para aumentar o acesso do público ao seu portal.

Como prova da crescente força do Big Brother Brasil na internet, a sétima edição do programa bateu todas as anteriores no mundo virtual. O mesmo se repetiu na TV paga, onde, em fevereiro de 2007, o Multishow alcançou a maior audiência da história da TV por assinatura no Brasil com 7,5 pontos (O GLOBO 13/03/2007). Somente no primeiro mês de exibição do programa em 2007, 13 milhões de pessoas visitaram a página virtual do $\mathrm{BBB}$, acessando os vídeos disponíveis 71 milhões de vezes, um aumento de $772 \%$ se comparado à edição anterior do reality show (O GLOBO, 19/02/2007).

Apesar de as organizações Globo não disponibilizarem dados quantitativos que nos permitam entender a contribuição do site do Big Brother Brasil para o portal Globo.com, nós podemos utilizar a ferramenta Google Trends - disponibilizada pelo site de busca Google -, para termos uma idéia deste movimento. Geramos um gráfico mostrando o volume comparativo de buscas dentro do Google no período de janeiro de 2004 a fevereiro de 2007, utilizando as expressões 'big brother brasil' (em azul) e 'globo.com' (em vermelho). Ao analisar a evolução das buscas podemos observar alguns dados eloqüentes. Em primeiro lugar, percebemos o enorme volume relativo de buscas pela expressão 'big brother brasil' exatamente durante o período de exibição do reality show, que tem sido produzido entre os meses de janeiro e abril de 
cada ano. Já quando analisamos o volume comparativo, percebemos um aumento das buscas pela expressão 'globo.com', aproximadamente na mesma proporção das buscas por 'big brother brasil' durante este mesmo período. Apesar do volume de buscas por 'globo.com' subir gradativamente ao longo dos anos, ele costuma aumentar mais quando o programa inicia, e sofrer uma queda logo depois do término do mesmo, como indica o gráfico. Esta proximidade das curvas vermelha e azul pode ser interpretada como evidência de que, enquanto alguns internautas utilizam a expressão 'big brother brasil' no Google para ter acesso à página do programa, outros usam a expressão 'globo.com' nesta ferramenta de busca, como forma de acesso ao portal da Globo, para, através deste, entrar na página do reality show.

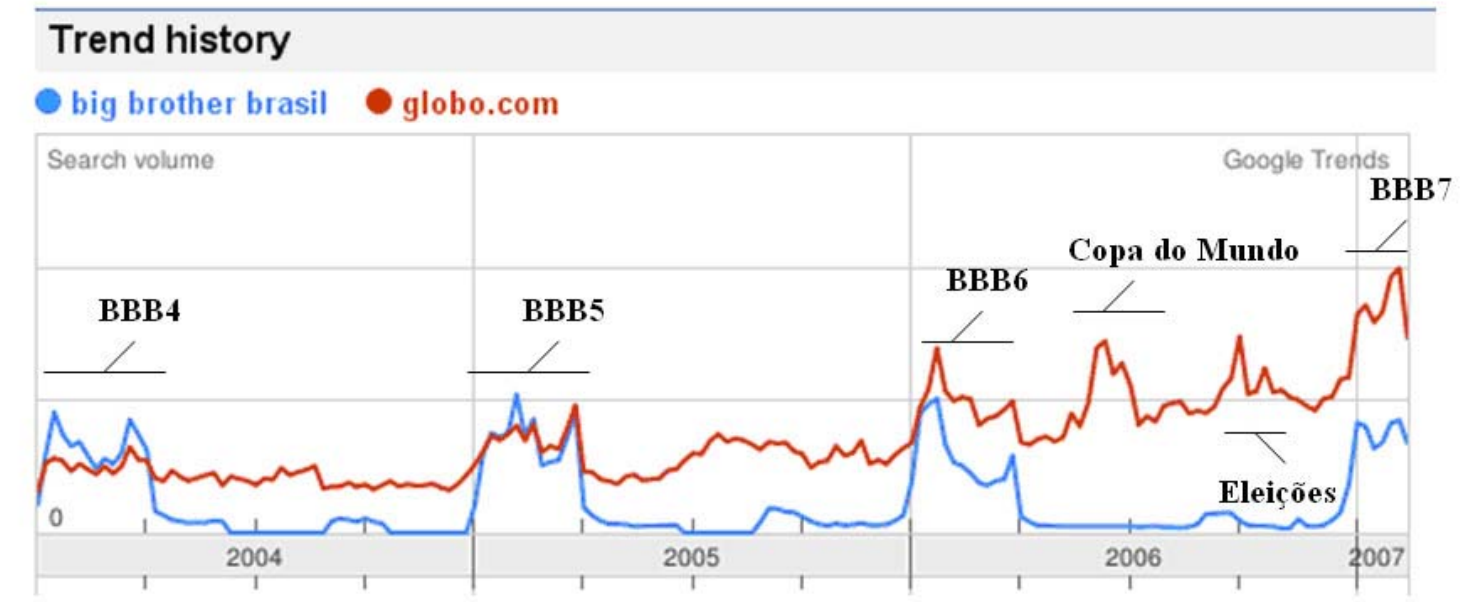

Fonte: Google Trends - http://www.google.com/trends

Como ilustração do peso do Big Brother Brasil como um evento midiático na internet, assinalamos dois outros eventos de grande relevância nacional no ano de 2006: a Copa do Mundo de futebol na Alemanha e as eleições gerais para cargos do executivo e legislativo. Somente este último evento foi capaz de gerar maior movimento no site Globo.com do que a sexta versão do Big Brother nacional (embora a base de internautas brasileiros no início daquele ano, quando o programa foi ao ar, fosse inferior àquela na época das eleições).

Estes dados deixam claro que o Big Brother não deve ter seu desempenho mercadológico avaliado somente pelos índices de audiência que este gera em 
televisão aberta. Durante o período de exibição do programa é comum acompanharmos a contínua divulgação de tais números pela imprensa como meio de avaliação de um eventual esgotamento ou não do formato do reality show. Entretanto, conforme sugere Bolaño (2005), a crescente popularização da TV paga e da internet no Brasil sinaliza uma diversificação no hábito do consumo midiático da população, o que levaria, ainda que de forma muito sutil, a um achatamento dos índices de audiência na TV aberta de um modo geral. De forma que, se quisermos avaliar o sucesso mercadológico do Big Brother Brasil de maneira correta, devemos levar em conta a sua evolução nas suas diferentes mídias de transmissão7. Portanto, os números apresentados acima indicam que a força deste evento midiático está na sua grande capacidade de ativar inúmeras mídias alternativas - em especial a internet -, e não somente no seu desempenho na TV aberta, mesmo que este esteja longe de ser desprezível ${ }^{8}$.

Assim como sugere Cosette Castro, a importância de novos formatos que consigam se adaptar em ambientes multimidiáticos é ainda maior se pensarmos na breve implantação da TV digital no Brasil. Dentro deste novo ambiente, o canal de televisão aberta que melhor conseguir produzir conteúdo dinâmico e interativo estará em clara vantagem em relação aos outros (CASTRO 2006).

\section{Novas Fontes de Receita}

A importância em reconhecer o Big Brother Brasil como um produto de natureza distinta de outros produtos midiáticos tradicionais é fundamental na medida em que este programa aponta para uma nova relação entre conteúdo, anunciante e consumidor. Ao contrário do modelo comercial tradicional onde as fronteiras entre estas três partes são bem delineadas, o aparecimento das novas mídias tem sinalizado o apagamento destes limites.

\footnotetext{
7 Infelizmente, não foi possível coletar números oficiais referentes ao uso da telefonia celular no Big Brother Brasil, ou mesmo do desempenho de vendas dos pacotes em pay-per-view. Entretanto, conversas informais do autor deste artigo com alguns dos responsáveis por estes números tenham deixado claro que as vendas da sétima edição do programa no Brasil, nestas mídias alternativas, superaram em muito as edições anteriores.

${ }^{8}$ Devido à confusão gerada por um certo desconhecimento por parte da imprensa na maneira de tratar os números de audiência referentes à TV aberta aferidos pelo IBOPE, encontramos índices, divulgados por jornais e outros meios de comunicação, muitas vezes contraditórios. Diferenças à parte, o Big Brother Brasil tem mantido, desde o seu início, índices médios na casa dos 40 pontos.
} 
Bill Gates afirma que o novo paradigma da publicidade em massa será a sua completa integração à programação de entretenimento. Ou seja, não será mais possível dissociar o que é conteúdo do que é campanha publicitária. Poderemos ter, por exemplo, um filme sendo exibido na televisão, ou mesmo na internet, no qual o ator usa um par de óculos de determinada marca que é oferecido on-line, enquanto o filme é exibido. Neste caso, o ator desempenharia dois papéis, um designado pelo diretor, com base na trama do roteiro, e outro de garoto propaganda, com base na necessidade do anunciante ${ }^{9}$ (GATES 1996).

O Big Brother parece ter levado este paradigma adiante. Na base da estrutura deste reality show, estão as provas de inteligência, resistência e sorte que os integrantes da casa são permanentemente submetidos. Nelas, eles disputam não somente prêmios dos patrocinadores, mas também posições hierárquicas privilegiadas dentro da casa (líder da semana, anjo etc.). Além destas provas, a versão brasileira do programa constantemente oferece eventos especiais para os participantes. Estes acontecimentos vão desde sessões de embelezamento, onde massagistas e esteticistas passam algumas horas dentro da casa tratando dos confinados, até apresentações de artistas de renome nacional e internacional, para os participantes. Em comum, tanto as provas, quanto os eventos, compartilham o interesse mercadológico da Globo em divulgar produtos de interesse comercial, sejam eles produtos cosméticos ou produtos culturais ${ }^{10}$.

A própria decoração interna da casa do Big Brother tem sua função econômica. Enquanto edições antigas do programa já tiveram os móveis fornecidos por empresas interessadas em expor e vender seus produtos, a edição de 2007 da versão brasileira aposta na divulgação de produtos do próprio grupo Globo. Dos três quartos temáticos usados pelos confinados para dormir, um foi baseado na 'TV Colosso', programa destinado ao público infantil exibido pela Rede Globo nos anos 90, e outro foi inspirado no filme 'Dois Filhos de Francisco', co-produzido pela Globo Filmes. Como conseqüência, a rotina dos participantes do Big Brother se mistura a

9 O Filme Truman Show oferece um bom exemplo de como isto poderia acontecer.

${ }^{10}$ Frequentemente, os produtos culturais exibidos no Big Brother pertencem à própria Globo, ou à empresas/ profissionais ligados ao grupo (ex. novas séries televisivas, apresentadores de programas da Globo, filmes lançados pela Globo Filmes etc). 
um merchandising contínuo, ao qual a audiência não tem como escapar, e até mesmo como identificar ${ }^{11}$.

Porém, além do anunciante, o Big Brother também integra o próprio consumidor ao conteúdo. Um dos grandes apelos do programa é a possibilidade oferecida à audiência de interferir nos rumos do mesmo. Esta interação acontece tanto na forma de tarefas a serem impostas aos confinados na casa ${ }^{12}$, como na eliminação semanal dos mesmos. Em ambos os casos, o público é quem determina o que deve ser feito ${ }^{13}$.

Também é importante lembrar que a página oficial na internet, assim como o portal de celular do reality show, foram pensados de maneira a acomodar discussões entre os fãs sobre os acontecimentos da casa. No site oficial, por exemplo, podemos encontrar chats, forums, e até mesmo links para blogs de fãs do Big Brother. De maneira semelhante, através do portal de celular pode-se conversar com diversos outros entusiastas do programa.

Em todos os casos acima, a audiência interfere na construção do produto a ser consumido. Mais do que isso, ao ajudar em sua construção, o está consumindo simultaneamente, seja pagando pela ligação do celular, na qual a Globo tem participação, seja gerando volume de acesso na página da internet, que é revertido em receita publicitária para o site.

Outra característica do Big Brother é a sua capacidade de gerar inúmeras fontes alternativas de receita. Além da tradicional venda de espaço publicitário e merchandising veiculados na TV Globo, podemos acrescentar as vendas de pacotes em pay-per-view, venda de espaço publicitário para o Multishow (que é realizada de forma independente das vendas da TV Globo), participação nas ligações e mensagens de texto feitas pela audiência através de parcerias com operadoras de telefonia celular e fixa, venda de espaço publicitário no site oficial, licenciamento de produtos, entre eles, o jogo para computador Big Brother Brasil, e as vendas de roupas e acessórios

\footnotetext{
${ }^{11}$ Esta prática já se tornou tão usual, que é simplesmente impossível avaliar quais produtos mostrados na casa fazem parte de alguma campanha de merchandising efetivamente, e quais não fazem.

${ }^{12}$ Chamado de Big Boss.

13 Na versão espanhola do programa o público escolhe até a condição climática durante a semana dentro da casa. Até chuva pode ser criada artificialmente no Big Brother espanhol.
} 
Big Brother feitas pelo site oficial. Não podemos deixar de mencionar os benefícios indiretos, como por exemplo: o aumento de assinantes na base NET, impulsionado pelas adesões de fãs que desejam comprar pacotes em pay-per-view - o que os obriga a serem assinantes de algum pacote NET - resultando em maior receita para os canais Globosat, uma vez que estes se beneficiam diretamente do aumento desta base.

Talvez, tão relevante quanto a receita financeira decorrente das diferentes formas de vender o programa, seja a capacidade do Big Brother de produzir novas celebridades a cada edição. Os seus produtores são responsáveis pela administração de uma verdadeira 'economia de celebridades', onde a transformação de pessoas ordinárias em 'produtos midiáticos' se torna um dos objetivos almejados pelo reality show. De maneira similar, os participantes buscam o status de celebridade como objetivo fim de sua participação no programa, e não como uma conseqüência natural (TURNER 2004). Na medida em que os participantes do Big Brother deixam a casa, eles participam de vários compromissos profissionais agendados pela Globo, com o objetivo de explorar ao máximo a fama recém adquirida por eles. Entre aparições em programas televisivos como, por exemplo, Ana Maria Braga ou Domingão do Faustão, até sessões de fotos para revistas ou sites pertencentes ao grupo, os exconfinados têm uma grande exposição em diferentes veículos midiáticos. Aqueles poucos que conseguem se manter em destaque na mídia por um tempo mais prolongado, acabam construindo carreiras artísticas na própria Globo, ou em outras empresas do ramo de comunicação. Isto significa que, mesmo com o término de cada edição do programa, os produtos resultantes dele ainda podem gerar dividendos por um longo período.

\section{Conclusão}

Quando estudamos a importância econômica de um programa como o Big Brother não podemos nos fixar somente nos lucros financeiros gerados por ele. Talvez tão importante, seja a sua capacidade de interagir com outros canais de veiculação e empresas do grupo detentor dos seus direitos.

No caso brasileiro, ainda acrescentaríamos um outro aspecto um pouco mais subjetivo: a renovação da grade da Rede Globo, representada pela exibição de um 
novo gênero televisivo mundial em horário nobre. Não podemos esquecer que em praticamente todos os países para onde o Big Brother foi vendido, os canais compradores eram estações de televisão em busca de um melhor posicionamento no seu mercado. Para tanto, estavam dispostos a correr riscos em novos e desconhecidos gêneros (BAZALGETTE 2005). No caso brasileiro a situação é completamente diferente, pois o comprador do seu formato, a Rede Globo, tem uma liderança incontestável de audiência, construída à custa de uma grade estável e baseada, fundamentalmente, em telenovelas com reconhecido padrão de qualidade (BORELLI 2005). No entanto, a fórmula das telenovelas começa a ser copiada com sucesso por canais concorrentes, representando perigo para a sua posição de destaque ${ }^{14}$.

Apesar de o Big Brother ter se adaptado ao Brasil de forma a incorporar diversos elementos da teledramaturgia em sua edição para TV aberta15 (O GLOBO 08/01/2007), ele significou uma mudança de peso para a Globo, tanto por ser coproduzido por uma empresa estrangeira, como pelo seu formato inovador e, por que não dizer, arriscado.

Prova disto, é que, mesmo já passados alguns anos desde a primeira edição do programa, ele continua recebendo críticas negativas em relação às suas características 'voyeurísticas' por parte da imprensa, que, frequentemente, o toma como símbolo da perda de qualidade do conteúdo televisivo atual, não escondendo um 'pânico moral' em relação ao reality show (BILTEREYST 2004; CURVELLO 2002; MATHIJS 2002). Entretanto, como bem observa Fredber \& Ollila, independente da pertinência de tais críticas, a audiência parece não se afetar por elas. Ao contrário, estas críticas acabam desempenhando um papel importante, ao darem mais visibilidade ao Big Brother, tornando-o ainda mais popular (OLLILA \& FREDBERG 2004).

O que fica claro é que este programa aponta para novos caminhos no relacionamento entre sociedade e conteúdo midiático. Portanto, para que se possa

\footnotetext{
14 Tanto o Sistema Brasileiro de Televisão (SBT), quanto a Rede Record estão fazendo grandes investimentos em seus núcleos de teledramaturgia nos últimos anos.

${ }_{15}$ Conforme mencionado anteriormente, diversos trabalhos ressaltam a capacidade do Big Brother de incorporar características culturais locais dos diferentes países onde ele é produzido (FRAU-MEIGS 2006; MADGER 2004; MATHIJS \& JONES 2004; ROSCOE 2004b).
} 
dar conta da complexidade deste fenômeno, é necessário analisa-lo sob diferentes perspectivas, seja do receptor, do próprio conteúdo em si, como do produtor. No Brasil, onde o Big Brother mantém uma posição de grande destaque no cenário midiático, o entendimento de sua posição dentro desta indústria se torna ainda mais urgente.

\section{Bibliografia}

ANDACHT, Fernando. Reality show Big Brother no Brasil e no Rio de la Plata: uma análise comparada de recepção. Revista Líbero, Ano VII, n. 13/14, 2004.

ANDREJEVIC, Mark. Reality TV: the work of being watched. Lanham, MD: Rowman \& Littlefield Publishers, Inc., 2003.

BAZALGETE, Peter. Billion dollar game. Londres: Time Warner Books, 2005.

BILTEREYST, Daniël. Big Brother and its moral guardians: reappraising the role of intellectuals in the Big Brother panic. In: MATHIJS, Ernest \& JONES, Janet (eds.). Big Brother International. Londres: Wallflower Paper, 2004.

BOLAÑO, César Ricardo Siqueira. Mercado brasileiro de televisão, 40 anos depois. In: BRITTOS, Valério Cruz \& BOLAÑO, César Ricardo Siqueira (orgs.). Rede Globo: 40 anos de poder e hegemonia. São Paulo, Editora Paulus, 2005.

BORELLI, Silvia. Telenovelas: padrão de produção e matrizes populares. In: BRITTOS, Valério Cruz \& BOLAÑO, César Ricardo Siqueira (orgs.). Rede Globo: 40 anos de poder e hegemonia. São Paulo, Editora Paulus, 2005.

BRATICH, Jack. "Nothing is left alone for too long". Reality programming and control society subjects. Journal of Communication Inquiry, Vol. 30, (1), p. 65-83, 2006.

CARTER, Gary. In front of our eyes: notes on Big Brother. In: MATHIJS, Ernest \& JONES, Janet (eds.). Big Brother International. Londres: Wallflower Paper, 2004.

CASTRO, Cosette. Por que os reality shows conquistam audiências? São Paulo: Editora Paulus, 2006.

CURVELLO, Vanessa. Big Brother Brasil: realidades espetaculares. Biblioteca on-line das Ciências da Comunicação, www.bocc.ubi.pt, 2002.

CORNER, John. Performing the real: documentary diversions. Television \& New Media, Vol. 3, n. 3, p.255-269, 2002.

COULDRY, Nick. Playing for celebrity: Big Brother as ritual event. Television \& New Media, Vol. 3, n. 3, p.283-293, 2002.

DUTTON, Edward C. Big Brother, pilgrimage and the Ndembu of Zambia: examining the Big Brother phenomenon through the anthropology of religion. The Journal of Religion and Popular Culture, Vol XII, 2006. 
FRAU-MEIGS, Divina. Big Brother and reality TV in Europe: towards a theory of situated acculturation by the media. European Journal of Communication, Vol. 21 (1), p.3356, 2006.

GATES, Bill. The road ahead. Nova York: Penguin, 1996.

GINDRE, Gustavo. Globo - o desafio da convergência. Revista de Economía Política de las Tecnologias de la Información e Comunicación, www.eptic.com.br, Vol. VII, n. 5, Maio/ Agosto 2005.

HILL, Annette. Big Brother: the real audience. Television \& New Media, Vol. 3, n. 3, Agosto, p. 323-340, 2002.

HOLMES, Su. 'But this time you choose!' Approaching the interactive audience in reality TV. International Journal of Cultural Studies, Vol. 7 (2), p. 213-231, 2004.

JONES, Janet. Show your real face: a fan study of the UK big brother transmissions. New Media \& Society. Vol. 5, p. 400-421, 2003.

. Emerging platform identities: Big Brother UK and interactive multi-platform usage. In: MATHIJS, Ernest \& JONES, Janet (eds.). Big Brother International. Londres: Wallflower Paper, 2004.

JOST, François. Loft Story: Big Brother France and the migration of genres. In: MATHIJS, Ernest \& JONES, Janet (eds.). Big Brother International. Londres: Wallflower Paper, 2004.

LAVENDER, Andy. Pleasure, performance and the Big Brother experience. Contemporary Theatre Review: An International Journal, vol. 13, no. 2, p. 15-23, May 2003.

MADGER, Ted. The end of TV 101: reality programs, formats, and the new business of television. In: MURRAY, Susan \& OUELLETTE, Laurie (eds.). Reality TV: Remaking Television Culture. New York: NYU Press, 2004.

MATHIJS, Ernest. Big Brother and critical discourse: the reception of Big Brother Belgium. Television \& New Media, Vol. 3, n. 3, p.311-322, 2002.

MATHIJS, Ernest \& JONES, Janet. Introduction: Big Brother international. In: MATHIJS, Ernest \& JONES, Janet (eds.).Big Brother International. Londres: Wallflower Paper, 2004.

MURDOCK, Graham. Marx on commodities, contradictions and globalisations - resources for a critique of marketised culture. Revista e-compós, Edição 7, dezembro 2006.

OLLILA, Sanne \& FREDBERG, Tobias. Setting stages: learning management from Big Brother. Fenix WP, n. 29, version 1, 2004.

PALMER, Gareth. Big Brother: an experiment in governance. Television \& New Media, Vol. 3 , n. 3, p.295-310, 2002.

RAPHAEL, Chad. The politic economic origins of reali-TV. In: MURRAY, Susan \& OUELLETTE, Laurie (eds.). Reality TV: Remaking Television Culture. New York: NYU Press, 2004.

ROSCOE, Jane. Multi-platform event television: reconceptualizing our relationship with television. The Communication Review, 7, p.363-369, 2004a.

Big Brother Australia: performing the 'real' twenty-four-seven. In: ALLEN, Robert \& HILL, Annette (eds.). The television studies reader. Londres: Routledge, p. 311-321, 2004b. 
STARRS, D. Bruno. Sara-Marie as feminist fairytale: from Big Brother to 'Big Sister'. Feminist Media Studies, Vol. 4, n. 3, p. 359-61, 2004.

TINCKNELL, Estella \& RAGHURAM, Parvati. Big Brother: reconfiguring the 'active' audience of cultural studies? European Journal of Cultural Studies, Vol. 5(2), p. 199-215, 2002.

THORNBORROW, J., \& MORRIS, D. Gossip as strategy: the management of talk about others on reality TV show "Big Brother". Journal of Sociolinguistics 8.2, P. 246(26), May 2004.

TURNER, Graeme. Understanding celebrity. Londres: Sage, 2004.

VAN ZOONEN, Liesbet. Desire and resistance: Big Brother and the recognition of everyday life. Media Culture \& Society, Vol. 23, p. 669-677, 2001. 